\title{
Plasma Cells in Chronic Endometritis are Easily Identified When Stained with Syndecan-1
}

Ilene B. Bayer-Garner, M.D., Soheila Korourian, M.D.

University of Arkansas for Medical Sciences, Little Rock, Arkansas

\begin{abstract}
Background: Chronic endometritis has been observed in $3-10 \%$ of women with irregular uterine bleeding who undergo endometrial biopsy. The diagnosis of chronic endometritis rests on the recognition of plasma cells in endometrial tissue that may show a prominent spindle cell stromal component, and is frequently difficult to date. Syndecan-1 is a cell-surface proteoglycan that is expressed on the cell surface of plasma cells. Design: Eighteen endometrial curettage cases with the diagnosis of chronic endometritis and 25 endometrial curettage cases of dysfunctional uterine bleeding, in females under the age of thirty-five in whom no other histopathologic changes were noted, were reviewed for the presence of plasma cells. Sections were then stained with syndecan-1. Results: All of the chronic endometritis cases showed easily visible syndecan-1 staining of plasma cell membranes. None of the cases of dysfunctional uterine bleeding showed presence of plasma cells in either the hematoxylin and eosin stained or syndecan-1 stained sections. Conclusions: In cases of suspected chronic endometritis in which no plasma cells can be found on hematoxylin and eosin stained slides, syndecan-1 may be an effective adjunct in the identification of plasma cells and thus aid in the diagnosis of chronic endometritis.
\end{abstract}

KEY WORDS: Syndecan-1, Chronic endometritis, Plasma cell.

Mod Pathol 2001;14(9):877-879

Chronic endometritis is a histopathologic entity that often shows superficial mucosal stromal edema, increased stromal density with a leukocytic infiltrate within both glands and stroma, and presence of plasma cells within the substance of the stroma (1). The diagnosis of chronic endometritis

Copyright $(2) 2001$ by The United States and Canadian Academy of Pathology, Inc.

VOL. 14, NO. 9, P. 877, 2001 Printed in the U.S.A.

Date of acceptance: May 1, 2001.

Address reprint requests to: Soheila Korourian, M.D., Professor, Department of Pathology, University of Arkansas for Medical Sciences, 4301 West Markham Street, Little Rock, AR 72205; KorourianSoheila@uams.edu. can be suspected when dyssynchronous maturation between the glands and stroma exists. Many investigators consider the presence of plasma cells in the endometrial stoma to be the diagnostic foundation of chronic endometritis (2). Chronic endometritis has been observed in 3-10\% of women with irregular uterine bleeding who undergo endometrial biopsy. It is not an uncommon finding in endometrial curettage specimens and in most cases a specific cause can be identified such as pelvic inflammatory disease, uterine leiomyomas, an intrauterine device, or postabortal and postpartum states (1-3). However, in some cases, the diagnosis of chronic endometritis is unexpected as the presenting complaint is nonspecific. In these cases in which no underlying cause can be found, the disorder is often self-limiting and the actual act of curettage may be therapeutic. Thus, although the diagnosis of chronic endometritis may at times be academic, it's greatest utility may be in establishing a cause of infertility or a cause of abnormal uterine bleeding (4).

Syndecan-1 is a cell surface marker that is expressed on the cell membrane of plasma cells. It has been extensively used to identify plasma cells in flow cytometry and is a reliable marker for quantifying both malignant and benign plasma cells in paraffin-embedded bone marrow biopsy specimens (5). Syndecan-1 is a member of a family of cell surface proteoglycans that mediates cell-cell adhesion, cell-extracellular matrix (ECM) adhesion, cell migration and proliferation (6-8). Syndecans bear predominantly heparan sulfate glycosaminoglycan chains and can bind via these heparan sulfate chains to both insoluble and soluble molecules within the ECM including interstitial collagens, fibronectin, tenascin, thrombospondin and growth factors such as FGF-2 (6). It is via these interactions that both normal and neoplastic cells invade the ECM. The ability to invade the ECM is important in that normal cells invade the ECM during wound healing, inflammation, and embryogenesis (9).

The ability of syndecan-1 to identify plasma cells by immunohistochemical means presented a unique opportunity to test a hypothesis. In cases 
suspicious for chronic endometritis in which no plasma cells could be identified by hematoxylin and eosin (H\&E) stains, immunohistochemical staining with syndecan-1 would identify plasma cells and thus aid in the diagnosis of chronic endometritis.

\section{MATERIALS AND METHODS}

Forty-three endometrial curettage cases that either had been given the diagnosis of chronic endometritis or had dysfunctional uterine bleeding but no histopathologic abnormalities could be identified were selected from the files at the University of Arkansas for Medical Sciences. The diagnosis of chronic endometritis was based on typical histologic features, that is, the recognition of plasma cells in endometrial tissue that showed a prominent spindle cell stromal component, and was frequently difficult to date. Eighteen of the endometrial curettage cases had been given the diagnosis of chronic endometritis. The twentyfive remaining endometrial curettage cases had been given the diagnosis of dysfunctional uterine bleeding and occurred in females under the age of thirty-five in whom no other histopathologic changes were noted. All biopsies were initially reviewed for the presence of plasma cells on standard H\&E stained slides. Specimens were fixed in $10 \%$ formalin and embedded in paraffin. Four-micron sections were cut and mounted on 3-aminopropyltrethoxy-silane-coated slides, dried, and deparaffinized before undergoing antigen retrieval by heat treatment in DAKO Target Retrieval solution (DAKO, Carpenteria, CA). The endogenous peroxidase activity was quenched with $0.3 \%$ peroxidase and nonspecific binding was quenched with horse serum block (Oncogene Research Products, Cambridge, MA). The DAKO Large Volume LSAB2 Alkaline Phosphatase Kit (DAKO) was used with 1:100 dilution of B-B4 (Serotec, Raleigh, NC), a mouse anti-human antibody that recognizes an epitope of human syndecan-1 (CD138) (5). Sections were incubated with biotinylated secondary antibody for thirty minutes, and then with streptavidin alkaline phosphatase for thirty minutes. The signal was visualized with DAKO Fast Red Substrate System (DAKO) yielding a red end product at the site of the target antigen. Positive control tissue consisted of normal skin in which the keratinocytes cell membrane immunoreactivity for syndecan-1 was evident in the basal layer and up through the granular layer. The negative control consisted of a 1:100 dilution of a nonspecific isotype-matched $\mathrm{IgG}_{1}$ mouse antibody, MCA 928 (Serotec), which was substituted for B-B4.

\section{RESULTS}

Samples of endometrium that showed features consistent with chronic endometritis and samples of endometrium from patients under the age of 35 that showed no histopathologic changes, ultimately diagnoses with dysfunctional uterine bleeding, were examined for presence of plasma cells both on $H$ \& E sections and on sections stained with syndecan-1.

All cases of chronic endometritis showed clearly visible syndecan-1 immunoreactivity on the plasma cell membranes (Fig. 1). This included classic plasma cells with the characteristic clockface chromatin in an eccentrically placed nucleus with a perinuclear halo, plasma cells that showed the classic nucleus but that was not eccentrically placed and did not show the perinuclear halo, and plasma cells in which only membrane and cytoplasm were visible. No other cells in the sections displayed immunopositivity for syndecan-1. None of the dysfunctional uterine bleeding cases showed presence of plasma cells in either the H\&E stained or syndecan-1 stained sections.

\section{DISCUSSION}

Syndecan-1 expression was examined in cases of chronic endometritis and in cases of dysfunctional

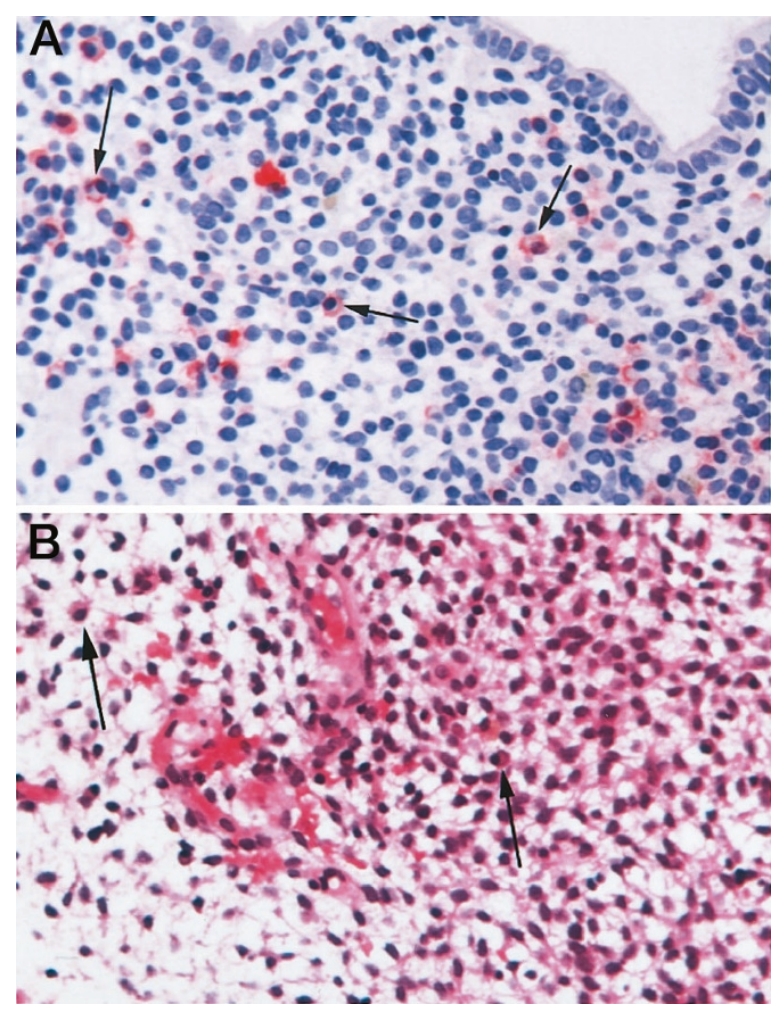

FIGURE 1. A, Plasma cells membrane staining with syndecan-1 in endometrial tissue showing a prominent spindle cell stromal component in chronic endometritis. B, H\&E staining of the same field showing plasma cells. 
uterine bleeding. All cases of chronic endometritis showed presence of plasma cells by light microscopic examination of both H\&E and syndecan-1 stained slides. Immunohistochemical staining with syndecan-1 enhanced the detection of plasma cells. It made the presence of classic plasma cells with the characteristic features of clock-face chromatin in an eccentrically placed nucleus with a perinuclear halo more visible, and it gave evidence that plasma cells that lacked some of the classic features were indeed plasma cells. It is in this second category of plasma cells that syndecan-1 immunopositivity may be the most advantageous. The search for plasma cells in the characteristic stroma of chronic endometritis can be very cumbersome. A number of conditions may mimic the histologic findings of chronic endometritis or interfere with the search for plasma cells. These include late menstrual or early proliferative endometrium, mononuclear inflammatory cell infiltrates, abundant stromal mitoses, stromal cell proliferation, the plasmacytoid appearance of stromal cells, or a pronounced predecidual reaction in a late secretory endometrium $(1,2)$. Syndecan-1 immunohistochemistry is specific for plasma cells. It is a reliable marker for quantifying plasma cells in paraffin-embedded bone marrow biopsy specimens $(5,6)$. It has also been noted to stain plasma cells in routine paraffinembedded skin biopsy sections (Bayer-Garner and Smoller, unpublished observation). This is important as plasmacytoid stromal cells and macrophages do not stain with syndecan-1, and plasma cell can be identified by immunohistochemical staining with syndecan- 1 in spite of conditions that may interfere with their identification on $H \& E$ slides.

The search for plasma cells, in order to diagnose chronic endometritis, does not always involve a cursory examination of the tissue. In many instances, it becomes a time consuming task. It is in cases that are suspicious for chronic endometritis, in which plasma cells cannot be identified in $\mathrm{H} \& \mathrm{E}$ sections, will syndecan-1 immunohistochemistry aid in identification of plasma cells and thus the diagnosis of chronic endometritis. Immunohistochemical staining with syndecan-1 will decrease the amount of time spent looking for plasma cells in cases suspicious for chronic endometritis, and will also be of assistance in diagnosing those cases in which other histological findings interfere with the search for plasma cells. It is important to note that relying on the mere presence of plasma cells to diagnose chronic endometritis is unreliable since plasma cells can be present in the endometrium in the absence of an inflammatory process (10). Only in the context of the characteristic milleaux of chronic endometritis should the diagnosis be considered and the identification of plasma cells be used for confirmation of the same.

In conclusion, in cases of suspected chronic endometritis in which no plasma cells can be found on a cursory examination of H\&E stained slides, and in those cases where other histologic findings interfere with the search for plasma cells, syndecan-1 may be an effective adjunct in the identification of plasma cells and thus aid in the diagnosis of chronic endometritis.

\section{REFERENCES}

1. Greenwood SM, Moran JJ. Chronic endometritis: morphologic and clinical observations. Obstet Gynecol 1981;58:17683.

2. Crum CP, Egawa K, Fenoglio CM, Richart RM. Chronic endometritis: the role of immunohistochemistry in the detection of plasma cells. Am J Obstet Gynecol 1983;147:812-5.

3. Korn AP, Hessol N, Padian N, Bolan G, Muzsnai D, Donegan E, et al. Commonly used diagnostic criteria for pelvic inflammatory disease have poor sensitivity for plasma cell endometritis. Sex Transm Dis 1995;22:335-41.

4. Vasudeva K, Thrasher TV, Richart RM. Chronic endometritis: a clinical and electron microscopic study. Am J Obstet Gynecol 1972;112:749-58.

5. Wijdenes J, Voous WC, Clement C, Post J, Morard F, Vita N, et al. A plasmocyte selective monoclonal antibody (B-B4) recognizes syndecan-1. Br J Haematol 1996;94:318-23.

6. Carey DJ. Syndecans: multifunctional cell-surface coreceptors. Biochem J 1997;327:1-16.

7. Ridley RC, Xiao H, Hata H, Woodliff J, Epstein J, Sanderson RD. Expression of syndecan-1 regulates human myeloma plasma cell adhesion to type I collagen. Blood 1993;81:76774 .

8. Liebersbach BF, Sanderson RD. Expression of syndecan-1 inhibits cell invasion into type-I collagen. J Biol Chem 1994; 268:20013-9.

9. Inki P, Joensuu H, Grenman R, Klemi P, Jalkanen M. Association between syndecan-1 expression and clinical outcome in squamous cell carcinoma of the head and neck. Br J Cancer 1994;70:319-23.

10. Brudenell JM. Chronic endometritis and plasma cell infiltration of the endometrium. J Obstet Gynaecol Br Emp 1955; 62:269-74. 